\title{
Abordagem da Omalgia nos Cuidados de Saúde Primários: Revisão da Literatura
}

\section{Approach to Shoulder Pain in Primary Health Care: Literature Review}

\author{
Rosana Pereira Dias ${ }^{1 *}$
}

*Autor Correspondente/Corresponding Author: Rosana Pereira Dias [rosana_p_dias@hotmail.com]

\section{RESUMO}

A omalgia é a terceira queixa musculoesquelética mais frequente nos cuidados de saúde primários (CSP). Este artigo pretende compreender a omalgia, a sua abordagem diagnóstica e terapêutica nos CSP. Realizou-se uma revisão narrativa. Identificaram-se 73 artigos na pesquisa e incluíram-se na revisão dados de 7 referências bibliográficas. A omalgia é mais frequente no sexo feminino, dos 45 aos 64 anos. Há pior prognóstico se idade avançada, sexo feminino, sintomatologia inicial intensa e associação a cervicalgia. As etiologias mais frequentes são: patologia da coifa dos rotadores, instabilidade e artrose gleno-umeral, capsulite adesiva e patologia da articulação acromioclavicular. Com uma anamnese completa a maioria das situações pode ser diagnosticada e gerida na primeira consulta, sem necessidade de investigação adicional. Quando necessária, a ecografia e a ressonância magnética nuclear são preferíveis. A referenciação a cuidados hospitalares deve ocorrer se queixas refratárias à terapêutica inicial (fármacos analgésicos e anti-inflamatórios e reabilitação física).

PALAVRAS-CHAVE: Cuidados de Saúde Primários; Dor de Ombro 


\section{ABSTRACT}

Shoulder pain is the third most frequent musculoskeletal complaint in primary health care (PHC). This article aims to understand shoulder pain, its diagnostic approach and therapy in PHC. A narrative review was carried out. Seventy-three articles were identified in the research and were included in the review data from 7 bibliographic references. Shoulder pain is more frequent in females, between 45 and 64 years old. There is worst prognosis if advanced age, female gender, intense initial symptoms, and association with neck pain. The most frequent etiologies are rotator cuff pathology, glenohumeral instability and arthrosis, adhesive capsulitis, and acromioclavicular joint pathology. With a complete clinical history and physical examination, most situations can be diagnosed and managed in a first visit without further investigation. If necessary, ultrasound and magnetic resonance imaging are preferable. Hospital referral should occur when complaints are refractory to the initial therapeutic approach (analgesic and anti-inflammatory drugs and physical rehabilitation).

KEYWORDS: Primary Health Care; Shoulder Pain

\section{INTRODUÇÃO}

O ombro é um complexo articular com um grande grau de mobilidade, permitindo o posicionamento do membro superior e movimentos em todos os planos. ${ }^{1}$

A omalgia é a terceira queixa musculoesquelética mais frequente de recurso aos cuidados de saúde primários (CSP), com uma prevalência estimada de 16\%-26\%, sendo uma das causas mais comuns de realização de tratamento de reabilitação. ${ }^{1-4}$ Se não tratada, é responsável por disfunção significativa, física e psicológica, má qualidade de vida e alta taxa de absentismo laboral, condicionando um importante impacto económico. ${ }^{1-3}$

O principal objetivo deste trabalho é compreender a omalgia, nomeadamente a abordagem diagnóstica e terapêutica das suas causas mais comuns nos CSP.

\section{MÉTODOS}

Este artigo consiste num trabalho de revisão narrativa.

A 31 de julho de 2021 realizou-se uma pesquisa, nas plataformas PubMed e UpToDate, tendo como discriminador os termos MeSH "shoulder pain" e "primary health care". Restringiu-se a pesquisa a meta-análises e revisões sistemáticas ou narrativas, publicadas entre janeiro de 2000 e julho de 2021 e escritas em inglês e português. Foram excluídos artigos de âmbito laboratorial ou animal, artigos que consistiam na descrição pormenorizada de técnicas diagnósticas ou terapêuticas, artigos que se referiam a outros sinais, sintomas e patologias distintas das do ombro, e artigos sem abstract ou texto completo disponível para análise. Posteriormente procedeu-se à leitura integral de artigos que não foram excluídos no passo anterior. Assim, foram analisados: a) cinco referências bibliográficas relevantes, identifi- cadas nos artigos previamente lidos na íntegra; b) dois livros sobre patologia reumatológica no âmbito dos cuidados de saúde primários em Portugal; c) três artigos tidos como relevantes para a elaboração deste trabalho, não abrangidos pelo método de pesquisa efetuado.

\section{RESULTADOS}

A Fig. 1 esquematiza o processo de pesquisa e seleção dos artigos incluídos nesta revisão.

Identificaram-se 71 artigos na pesquisa e incluíram-se na revisão, dados referentes a 7 referências bibliográfcas. De seguida, serão apresentados os dados pertinentes no que diz respeito à anatomia, epidemiologia, anamnese e exame objetivo na omalgia, bem como principais síndromes clínicas associadas a omalgia e a sua abordagem diagnóstica e terapêutica e critérios de referenciação para cuidados hospitalares.

\section{ANATOMIA}

O complexo articular do ombro é constituído por um conjunto de pontos de mobilidade articular e planos de deslizamento miofascial. Do ponto de vista anatómico, o ombro apresenta três articulações verdadeiras: a articulação esterno-clavicular, a acrómio-clavicular e a gleno-umeral, as quais estão associadas a dois planos de deslizamento/articulações funcionais, a escapulo-torácica e o arco subdeltoideu. ${ }^{1,2}$

As estruturas do ombro são a origem e inserção de um elevado número de músculos que atuam em sinergia e possibilitam a mobilidade do membro superior. ${ }^{1}$ Por exemplo, no que se refere à coifa dos rotadores, esta é constituída por quatro músculos, o supraespinhoso, o infraespinhoso, o redondo menor e o subescapular, e a sua principal função é a de estabilização primária e 


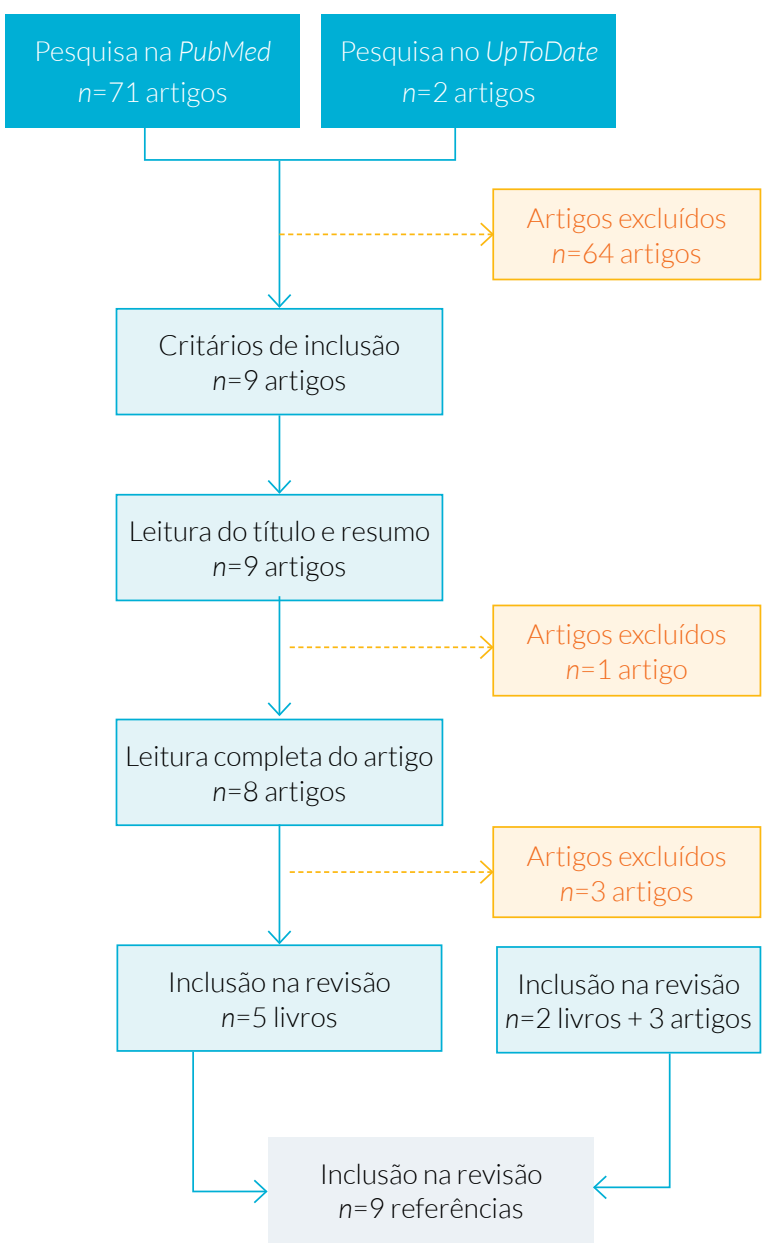

$n$ - número.

FIGURA 1. Esquematização do processo de pesquisa e seleção dos artigos incluídos nesta revisão.

coaptação da articulação gleno-umeral. O músculo supraespinhoso tem origem na fossa supraespinhosa da omoplata e insere-se na grande tuberosidade do úmero; tem como função a realização do $15^{\circ}$ iniciais de abdução do membro superior, em colaboração com o músculo deltoide. $\bigcirc$ músculo infraespinhoso tem origem na fossa infraespinhosa da omoplata e insere-se também na grande tuberosidade do úmero, mas na região mais posterior, fundindo-se na região póstero-superior com a inserção do tendão do músculo supraespinhoso; é o principal rotador lateral do ombro. $\bigcirc$ músculo redondo menor tem origem na porção póstero-inferior da omoplata e insere-se inferiormente ao tendão do músculo infraespinhoso; para além de rotador lateral do ombro, auxilia na sua adução. Por fim, o músculo subescapular tem origem na fossa subescapular da omoplata e insere-se na pequena tuberosidade umeral, auxiliando ainda na adução. ${ }^{1}$

A biomecânica articular complexa do ombro é um sistema de rotações que se inicia na clavícula, passa pela omoplata, como base de suporte do braço e culmina no úmero. Ao nível da omoplata, podemos definir 3 movimentos isolados, com relevância em termos funcionais no seu apoio ao movimento do ombro: a rotação interna e externa, a rotação superior e inferior, e o tilt anterior e posterior (inclinação escapular com afastamento ou aproximação do ângulo inferior da omoplata à parede torácica). Estes movimentos estão dependentes do balanço entre duas forças iguais em magnitude, mas em direções opostas que, em equilíbrio, estabilizam e mobilizam a omoplata (denominados pares funcionais ou force-couples). Ou seja, a rotação externa e interna da omoplata associa-se ao serreado anterior e trapézio médio/ romboides; por outro lado, a combinação de forças de vetor oposto do trapézio superior e serreado anterior permite a rotação superior da omoplata, movimento de extrema importância no ritmo escapulo-umeral; por fim, o movimento de tilt depende da combinação de forças do trapézio inferior e peitoral menor. ${ }^{1}$ Assim se compreende que, a ativação dos músculos escapulo-torácicos não sendo síncrona, deverá ocorrer num ritmo coordenado com a articulação gleno-umeral, chamado ritmo escapulo-umeral. Quando tal não acontece, surge uma condição conhecida como discinesia escapulo-torácica, definida como uma alteração na posição em repouso ou na mobilidade escapular, vista mais frequentemente como uma disfunção do ombro, em vez de uma patologia em si mesma. ${ }^{1,5}$

É esta conjugação de movimentos e da dinâmica muscular que permite movimentar a mão com uma amplitude superior a todas as articulações do corpo humano.,

\section{EPIDEMIOLOGIA}

A omalgia tem uma incidência de 15 episódios por 1000 utente, com uma prevalência de $70 \%$ ao longo da vida, na população geral. Estima-se que 25\% dos doentes com omalgia já tiveram episódios semelhantes anteriormente e desses, a maioria apresentou dor persistente ao longo de 1 ano. ${ }^{1,6}$

A omalgia é mais frequente no sexo feminino, entre os 45 e os 64 anos. Na população mais jovem, a patologia do ombro é desencadeada principalmente por atividades desportivas, nomeadamente aquelas que exijam movimentos repetidos acima do nível da cabeça (por exemplo, natação ou voleibol). Na população mais idosa, as lesões devem-se a atividades diárias (recreativas ou profissionais) com movimentos repetitivos., ${ }^{1,2}$ Assim se compreende que ocorre uma incidência aumentada de diferentes patologias por grupos etários: nos jovens as mais representativas são as lesões labrais, os conflitos e as roturas parciais articulares; na meia-idade ocorrem sobretudo tendinopatias calcificantes e capsulites ade- 
sivas; e nos mais idosos surgem as roturas degenerativas da coifa e a omartrose..$^{1,2}$

A patologia do ombro pode ter um curso autolimitado (como por exemplo, na tendinopatia simples ou calcificante, que se devem a alterações nos feixes tendinosos - hiperplasia fibroblástica, neovascularização, espessamento ou alterações na matriz do colagénio, ou deposição intratendinosa de cristais de fosfato de cálcio) ou tornar-se crónica, com evolução desfavorável quer em termos de gravidade, quer de extensão da doença (como por exemplo, na patologia da coifa dos rotadores, que compreende um espectro de alterações patológicas, desde a tendinose degenerativa às roturas tendinosas parciais ou totais; ou na omartrose, que pode ser uni ou bilateral, de etiologia primária/essencial ou secundária). ${ }^{1} \mathrm{O}$ pior prognóstico associa-se a idade avançada, sexo feminino, sintomatologia inicial intensa e associação a cervicalgia. ${ }^{4}$

\begin{abstract}
ANAMNESE
A história clínica deve ser minuciosa, dado ter implicações diagnósticas e terapêuticas. ${ }^{6}$ Além do motivo da consulta, deve-se determinar a idade, o género, o membro superior dominante, a profissão e/ou prática desportiva, antecedentes médicos e cirúrgicos (incluindo trau-
\end{abstract}

TABELA 1. Testes para avaliação do ombro., 1,2,7,8

\begin{tabular}{|c|c|c|c|}
\hline NOME & IMPORTÂNCIA & TÉCNICA PARA EXECUÇÃO & SIGNIFICADO \\
\hline Teste de Jobe & $\begin{array}{l}\text { Específico para avaliação } \\
\text { do tendão do músculo su- } \\
\text { praespinhoso. }\end{array}$ & $\begin{array}{l}\text { Elevação do ombro a } 90^{\circ} \text { e angulação a } 30^{\circ} \mathrm{com} \\
\text { o plano frontal, alinhando o úmero com o plano } \\
\text { da omoplata; com o ombro em rotação medial, } \\
\text { apontando o polegar para baixo, o examinador } \\
\text { aplica força para baixo contra resistência. }\end{array}$ & $\begin{array}{l}\text { Teste reativo se a manobra despoletar dor e } \\
\text { positivo se o doente ceder à resistência exer- } \\
\text { cida. }\end{array}$ \\
\hline Palm Up Test & $\begin{array}{l}\text { Específico para patologia } \\
\text { do tendão da longa porção } \\
\text { do bicípite braquial. }\end{array}$ & $\begin{array}{l}\text { Cotovelo do doente em extensão e palma da } \\
\text { mão voltada para cima; o examinador exerce re- } \\
\text { sistência contra a elevação anterior do ombro. }\end{array}$ & $\begin{array}{l}\text { Teste positivo quando é desencadeada dor, } \\
\text { referida ao trajeto deste tendão. }\end{array}$ \\
\hline Teste de Patte & $\begin{array}{l}\text { Avalia a integridade do } \\
\text { tendão infraespinhoso. }\end{array}$ & $\begin{array}{l}\text { Ombro do doente a } 90^{\circ} \text { de elevação lateral e } \\
\text { cotovelo em flexão de } 90^{\circ} \text {; doente faz rotação } \\
\text { externa contra resistência exercida pelo exami- } \\
\text { nador. }\end{array}$ & $\begin{array}{l}\text { Teste positivo se o doente não for capaz de } \\
\text { realizar rotação externa ou se apresentar dor } \\
\text { exacerbada (pode indicar rotura ou tendino- } \\
\text { patia, respetivamente). }\end{array}$ \\
\hline Lift Off Test & $\begin{array}{l}\text { Avalia a integridade do } \\
\text { tendão do músculo subes- } \\
\text { capular. }\end{array}$ & $\begin{array}{l}\text { Doente coloca a mão na região dorsal, com o } \\
\text { dorso da mão voltado para a frente; o examina- } \\
\text { dor pede ao doente para fazer força posterior } \\
\text { (afastar a mão do dorso) contra resistência. }\end{array}$ & Teste positivo quando despoleta dor. \\
\hline \multirow[t]{2}{*}{ Arco doloroso } & & \multirow{2}{*}{$\begin{array}{l}\text { O ombro é estabilizado com uma mão do exa- } \\
\text { minador, enquanto o membro superior é abdu- } \\
\text { zido. }\end{array}$} & $\begin{array}{l}\text { Dor entre } 60^{\circ}-120^{\circ} \text { de amplitude indica con- } \\
\text { flito subacromial. }\end{array}$ \\
\hline & & & $\begin{array}{l}\text { Dor }>120^{\circ} \text { de amplitude indica patologia da } \\
\text { articulação acromioclavicular. }\end{array}$ \\
\hline Teste de Hawkins & $\begin{array}{l}\text { Deteção do conflito suba- } \\
\text { cromial. }\end{array}$ & $\begin{array}{l}\text { Com o ombro elevado a } 90^{\circ} \text { no plano da omo- } \\
\text { plata e com o cotovelo em flexão, o examinador } \\
\text { faz rotação medial do ombro (diminuindo o es- } \\
\text { paço subacromial. }\end{array}$ & Teste positivo quando despoleta dor. \\
\hline Teste de Neer & $\begin{array}{l}\text { Deteção do conflito suba- } \\
\text { cromial. }\end{array}$ & $\begin{array}{l}\text { Com o membro superior em pronação e a omo- } \\
\text { plata estabilizada, o primeiro é fletido. }\end{array}$ & Teste positivo quando há dor. \\
\hline Teste de Yergason & $\begin{array}{l}\text { Avalia alterações na longa } \\
\text { porção do bicípete ou no } \\
\text { labrum glenoideu. }\end{array}$ & $\begin{array}{l}\text { Com o membro superior ao longo do corpo e } \\
\text { com o antebraço fletido a } 90^{\circ} \text {, o antebraço faz } \\
\text { supinação contra resistência. }\end{array}$ & $\begin{array}{l}\text { A existência de dor indica inflamação na longa } \\
\text { porção do bicípete ou lesões no labrum gle- } \\
\text { noideu. }\end{array}$ \\
\hline Drop arm test & $\begin{array}{l}\text { Avalia alterações na coifa } \\
\text { dos rotadores. }\end{array}$ & $\begin{array}{l}\text { O membro superior é levado passivamente a } \\
\text { abdução total; depois o doente tenta a adução. }\end{array}$ & $\begin{array}{l}\text { Incapacidade em aduzir indica rotura da coifa } \\
\text { dos rotadores. }\end{array}$ \\
\hline Cross arm test & $\begin{array}{l}\text { Específico para patologia } \\
\text { da articulação acromiocla- } \\
\text { vicular. }\end{array}$ & $\begin{array}{l}\text { Com o cotovelo em extensão, faz-se adução } \\
\text { horizontal do ombro a } 90^{\circ} \text { de elevação anterior. }\end{array}$ & $\begin{array}{l}\text { Teste positivo se dor localizada na articulação } \\
\text { acromioclavicular. }\end{array}$ \\
\hline Teste de O’Brien & $\begin{array}{l}\text { Específico para patologia } \\
\text { do labrum e da articulação } \\
\text { acromioclavicular. }\end{array}$ & $\begin{array}{l}\text { Com o cotovelo em extensão completa, o doen- } \\
\text { te faz elevação anterior até } 90^{\circ} \text { com rotação } \\
\text { medial, contra resistência. Posteriormente o } \\
\text { teste é repetido em rotação lateral (polegar a } \\
\text { apontar para cima). }\end{array}$ & $\begin{array}{l}\text { Teste positivo quando despoleta dor ou clique } \\
\text { na rotação medial, com redução ou ausência } \\
\text { de dor na rotação lateral: } \\
\text { • se dor superficial na articulação acromiocla- } \\
\text { vicular, suspeitar de lesão nesta articulação; } \\
\text { - se dor mais profunda e generalizada, suspei- } \\
\text { tar de lesão do labrum. }\end{array}$ \\
\hline $\begin{array}{l}\text { Teste de } \\
\text { apreensão e } \\
\text { recolocação }\end{array}$ & $\begin{array}{l}\text { Específico para instabili- } \\
\text { dade da articulação gleno- } \\
\text {-umeral. }\end{array}$ & $\begin{array}{l}\text { Doente em decúbito dorsal, com o cotovelo a } \\
90^{\circ} \text { e o ombro em elevação lateral a } 90^{\circ} \text {, sendo } \\
\text { realizada rotação lateral do ombro. Caso haja } \\
\text { sensação de apreensão/instabilidade, repete- } \\
\text {-se com o examinador a colocar a mão sobre a } \\
\text { região anterior do ombro, para o doente não } \\
\text { sentir a apreensão/instabilidade. }\end{array}$ & $\begin{array}{l}\text { Teste positivo quando o doente sente ins- } \\
\text { tabilidade na primeira manobra, mas não na } \\
\text { segunda. }\end{array}$ \\
\hline
\end{tabular}


TABELA 2. Classificação etiológica da omalgia. ${ }^{1,2}$

\begin{tabular}{l} 
PATOLOGIA INTRÍNSECA \\
\hline Patologia Periarticular \\
\hline - Tendinopatia da coifa \\
\hline - Patologia do tendão da longa porção do bicípite braquial \\
- Síndrome do conflito subacromial \\
\hline - Patologia da bursa subacromial, subdeitoideia, subcoroideia \\
- Instabilidade não traumática \\
\hline Patologia Articular \\
\hline - Omartrose \\
\hline - Capsulite adesiva \\
- Artrose da articulação acromioclavicular \\
\hline Traumatologia \\
- Fratura da clavícula \\
- Fratura do úmero proximal \\
- Luxação da gleno-umeral e instabilidade articular traumática \\
\hline - Luxação acromioclavicular \\
\hline PATOLOGIA EXTRíNSECA \\
\hline Origem Visceral \\
\hline - Cardiovascular \\
- Pulmonar \\
- Abdominal \\
- Patologia Maligna \\
- Oríndrome do desfiladeiro torácico \\
\hline - Síndrome doloroso regional complexo \\
\hline
\end{tabular}

máticos) e a medicação habitual. . $^{1-4,7}$ Importa lembrar que a patologia traumática é mais frequente em jovens, enquanto a patologia degenerativa é mais comum nos idosos. Antecedentes cirúrgicos do ombro aumentam a probabilidade de capsulite adesiva ou osteoartrose. A história médica de doenças reumatológicas alerta para as artrites inflamatórias. ${ }^{6}$

Na caracterização da omalgia, importa definir a duração dos sintomas e o seu início (agudo ou insidioso), descrever o mecanismo lesional, no caso de traumatismo, caracterizar os sintomas associados (por exemplo, equimose imediata, edema, deformidade, assimetria, amiotrofia), o tipo de dor (lancinante, moedeira, picada) e os fatores de alívio ou agravamento., ${ }^{1,2,7} \mathrm{O}$ ritmo dos sintomas (mecânico ou inflamatório) é um dos aspetos mais relevantes. Se houver predomínio das queixas ao final do dia e relacionadas com o esforço/progressão da atividade desportiva/profissional/lazer, com melhoria após o repouso, a dor é considerada mecânica. ${ }^{1,3}$ Se o predomínio das queixas for noturno ou matinal, com despertares noturnos pela dor, com rigidez matinal, a dor é inflama- tória. Quando ambos os componentes estão presentes, a dor é de características mistas. ${ }^{1}$ Por exemplo, quando falamos das tendinopatias da coifa dos rotadores ou da longa porção do bicípite braquial e da patologia da bursa subacromial, subdeltóideia ou subcoracóideia, as queixas álgicas podem ocorrer apenas associadas a esforços (dor mecânica) ou podem surgir em repouso, com agravamento noturno, sobretudo quando na fase inflamatória (dor inflamatória); o mesmo ocorre no conflito subacromial, onde as queixas álgicas agravam com os movimentos e em carga, podendo ocorrer também dor noturna (dor de características mistas). Outro exemplo, a instabilidade não traumática do ombro surge como uma dor mais mecânica, dado ser desencadeada sobretudo com atividades de elevação lateral e rotação externa do ombro. No caso da omartrose, a dor pode ser de natureza mecânica ou de natureza mista nas situações de agudização da patologia osteoarticular. Na capsulite adesiva, na fase de maior dor (fase dolorosa ou inflamatória), esta é tipicamente inflamatória, sendo particularmente pronunciada à noite. ${ }^{1}$

É ainda importante compreender o impacto da omalgia na qualidade de vida do doente (na atividade desportiva, na qualidade do sono, nas atividades básicas do quotidiano, recreativas ou profissionais) e procurar fatores predisponentes, como erros posturais ou descondicionamento físico, com especial atenção para os atletas no que respeita a excesso de carga nos treinos ou erros na execução do gesto técnico., ${ }^{1,2}$

Além disto, deve-se prestar atenção a alguns "red flags":

- antecedentes de cancro, sinais ou sintomas sugestivos de doença neoplásica (edema, tumefações ou deformidades de novo e sem etiologia conhecida);

- eritema, febre, mau-estar geral, queixas respiratórias;

- trauma, deformidade do membro, limitação da mobilidade articular, dor intensa

- défices motores e sensitivos significativos sem etiologia conhecida. ${ }^{3,4}$

Assim, uma anamnese cuidada é uma ferramenta diagnóstica para a maioria das etiologias de omalgia, intrínsecas (patologia da coifa dos rotadores, patologia gleno-umeral, patologia da articulação acromioclavicular, infeção ou fratura/luxação traumática) ou extrínsecas (irritação diafragmática, pneumonia, tumores apicais do pulmão, enfarte do miocárdio, metástases, radiculopatia cervical).1,2,4

\section{EXAME OBJETIVO}

O exame objetivo permite identificar a existência de patologia, medir e monitorizar o grau de disfunção, promover um programa de reabilitação personalizado às 
necessidades do doente e aumentar a confiança deste no processo terapêutico. ${ }^{3} \mathrm{O}$ exame objetivo deve incluir a inspeção (avaliação de assimetrias, atrofia muscular e deformidades evidentes), palpação e a mobilidade ativa e passiva do ombro, complementados com testes específicos (Tabela 1).1,2,7,8 A combinação destes fatores, reduz o leque de diagnósticos diferenciais.

\section{PRINCIPAIS SÍNDROMES CLÍNICAS E A SUA ABORDAGEM DIAGNÓSTICA E TERAPÊUTICA}

As causas de omalgia podem ser divididas em 2 grandes grupos etiológicos: intrínsecos (a causa está no próprio ombro) e extrínsecos ou dor referida (Tabela 2). A patologia intrínseca pode ter origem articular ou periarticu-

TABELA 3. Abordagem diagnóstica e terapêutica das principais etiologias intrínsecas periarticulares de omalgia. 1,2,6,7,9

\begin{tabular}{|c|c|c|c|}
\hline PATOLOGIA & OBSERVAÇÕES & $\begin{array}{l}\text { MEIOS COMPLEMENTARES } \\
\text { DE DIAGNÓSTICO }\end{array}$ & TRATAMENTO \\
\hline \multicolumn{4}{|c|}{ PATOLOGIA INTRÍNSECA } \\
\hline
\end{tabular}

- Tendinopatia

da coifa dos

rotadores

Responsável por 65\%-70\% das consultas por omalgia nos CSP. Abrange: tendinose degenerativa, tendinopatia simples e calcificada e roturas. $\mathrm{O}$ tendão do supraespinhoso é o mais afetado.

A ECO é um excelente exame e apresenta acuidade diagnóstica semelhante à RM. A artro-RM tem maior sensibilidade e especificidade para roturas parciais tendinopatia do subescapular. A Rx é importante no diagnóstico de tendinopatia calcificada.

\section{A TC é poucas vezes usada.}

Tendinopatia calcificante da coifa dos rotadores

É uma das causas mais frequentes de morbilidade do ombro e é responsável por $10 \%$ das consultas por omalgia. Afeta sobretudo mulheres entre os 30 e os 50 anos.

Patologia do TLPBB

É difícil de distinguir da tendinopatia da coifa, sendo que estas entidades podem ocorrer concomitantemente.

\section{Síndrome \\ do conflito subacromial}

("shoulder impingement syndrome")

\section{- Patologia} da bursa subacromial, subdeitoideia, subcoroideia
Na população geral associa-se a alterações posturais (coluna vertebral e cintura escapular), gestos repetidos ou posicionamentos incorretos na atividade laboral, ausência de exercício físico ou incorreta execução técnica de exercícios desportivos.

Pode apresentar quadro clínico semelhante ao da tendinopatia da coifa.
A Rx simples ântero-posterior em posição neutra com rotação interna e externa é habitualmente suficiente para o diagnóstico. A ECO tem a mesma sensibilidade que a Rx. O estudo doppler é útil para prever a evolução da patologia e da dor. A RMN raramente está indicada, sendo mais usada em doentes com dor refratária, para identificar complicações (exemplo: rotura tendinosa).

A Rx não está indicada. A ECO é o exame usado com maior frequência, com resultados semelhantes à RM no diagnóstico de roturas totais, luxação e subluxação. A artro-RM tem maior sensibilidade diagnóstica.

É clínico. Os exames de imagem (ECO e RM) são relevantes na confirmação diagquelas provocadas pelo conflito.

A ECO é o exame recomendado. nóstica, identificação de etiologia e de se-

Na maioria dos casos é TxC (evicção de movimentos causais, uso de analgésicos, AINEs e/ ou relaxantes musculares). O TxF e as infiltracões locais com ou sem corticosteroide podem ajudar no alívio álgico e melhoria da funcionalidade. A infiltração de produtos ortobiológicos (por exemplo: plasma rico em plaquetas) tem pouca evidência. $\bigcirc \mathrm{TxCx}$ (habitualmente artroscópico) deve ser reservado para as situações refratárias.

As calcificações em fase de reabsorção (fase mais dolorosa) têm prioridade terapêutica. 0

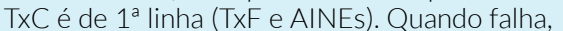
há outras opções para resolver os sintomas e evitar o TxCx; estas são: ondas-choque, barbotage ecoguiada e infiltração local com corticoides. Se falência destas duas linhas terapêuticas por mais de 6 meses, o TxCx, sobretudo artroscópico, é uma opção.

Tratamento inicial é TxC (modificação das atividades diárias, medicação analgésica e anti-inflamatória) e TxF. O TxCx não está indicado na tendinopatia do TLPBB isolado, mas está perante falência do TxC, sendo frequentemente astroscópico.

É primariamente TxC: TxF para correção do ritmo escapulo-umeral e escapulo-torácico por 3-6 meses, com controlo álgico e inflamatório farmacológico. A infiltração cortico-anestésica está indicada em casos muito sintomáticos e com grande limitação funcional. O TxCx está direcionado para a resolução das patologias na base da síndrome do conflito subacromial.

O tratamento inicial é TxC (crioterapia, tratamento farmacológico analgésico e anti-inflamatório e evicção dos fatores predisponentes). $\bigcirc$ TxF também pode estar indicado. Na omalgia intensa e incapacitante ou renitente a infiltração local com corticosteroides poderá ser usada. Nos poucos casos sintomáticos refratários, o TxCx com descompressão subacromial poderá estar indicado.

Na maioria das vezes é TxC (modificação das atividades causais, técnicas para analgesia e um programa intensivo de reabilitação para manutenção/restauração do movimento funcional completo, fortalecimento muscular e treino propriocetivo). Quando os episódios de luxação são frequentes ou no caso de atletas e jovens poderá ser indicado TxCx.
É clínico, servindo os exames de imagem para identificar possíveis variantes anatómicas e causas patológicas. A TC é habitualmente usada para planeamento pré-operatório. A ECO serve para exclusão de patologia da coifa associada. A RM é o exame recomendado na instabilidade gleno-umeral não traumática porque diagnostica as suas principais causas.

\section{- Instabil traumática}

AINE - Anti-inflamatório não esteroide; ECO - Ecografia; RM - Ressonância magnética; Rx - Radiografia; TC - Tomografia computorizada; TLPBB - Tendão da longa porção do bicípite braquial; TxC - Tratamento conservador; TxCx - Tratamento cirúrgico; TxF - Tratamento fisiátrico. 
TABELA 4. Abordagem diagnóstica e terapêutica das principais etiologias intrínsecas articulares de omalgia. 1,2,6,7,10

\begin{tabular}{|c|c|c|c|}
\hline PATOLOGIA & OBSERVAÇÕES & $\begin{array}{l}\text { MEIOS COMPLEMENTARES } \\
\text { DE DIAGNÓSTICO }\end{array}$ & TRATAMENTO \\
\hline \multicolumn{4}{|c|}{ PATOLOGIA INTRÍNSECA } \\
\hline \multicolumn{4}{|l|}{ Patologia Articular } \\
\hline - Omartrose & $\begin{array}{l}\text { Doença degenerativa; causa de } \\
\text { omalgia em } 2 \%-5 \% \text { dos casos. Os } \\
\text { sintomas mais comuns são: dor e } \\
\text { limitação da amplitude articular do } \\
\text { ombro nas AVDs. }\end{array}$ & 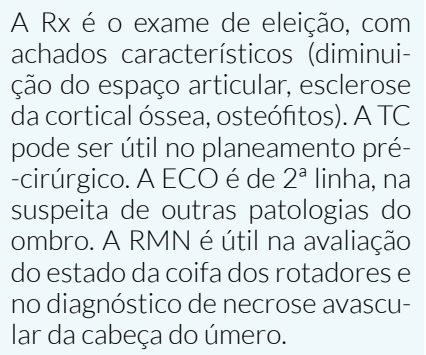 & $\begin{array}{l}\text { A maioria dos doentes responde ao TxC (mo- } \\
\text { dificação da atividade, fisioterapia, controlo } \\
\text { álgico farmacológico). São opções: infiltração } \\
\text { corticoanastésica ecoguiada intra-articular na } \\
\text { fase aguda e visco-suplementação com ácido } \\
\text { hialurónico na fase crónica. Suplementos de } \\
\text { condroitina ou glucosamina carecem de evidên- } \\
\text { cia científica. OTxCx está reservado na falência } \\
\text { dos anteriores. }\end{array}$ \\
\hline $\begin{array}{l}\text { - Capsulite adesiva } \\
\text { (“ombro congelado") }\end{array}$ & $\begin{array}{l}\text { O membro superior não dominante } \\
\text { é ligeiramente mais afetado. Exis- } \\
\text { tem alguns FR ( }>50 \text { anos; trauma do } \\
\text { ombro; imobilização prolongada do } \\
\text { membro superior; cirurgia prévia; } \\
\text { diabetes mellitus, hipo e hipertiroi- } \\
\text { dismo; doenças autoimunes; doença } \\
\text { de Parkinson; AVC). Curso da doen- } \\
\text { ça: fase dolorosa ou inflamatória } \\
\text { (2-9 meses); fase de congelamento } \\
\text { ou rigidez (4-12 meses); fase de re- } \\
\text { cuperação ou descongelamento (5- } \\
24 \text { meses). Vários doentes reportam } \\
\text { sequelas, perdendo de forma defini- } \\
\text { tiva 15\% da mobilidade do ombro. }\end{array}$ & $\begin{array}{l}\text { É clínico. A Rx ou a ECO são para } \\
\text { exclusão de outras causas de } \\
\text { omalgia. Perante um exame obje- } \\
\text { tivo inconclusivo, a RM ou a artro- } \\
\text {-RM podem ser consideradas. }\end{array}$ & $\begin{array}{l}\text { A abordagem terapêutica não é consensual. } \\
\text { Na fase dolorosa ou inflamatória: pode incluir } \\
\text { tratamento farmacológico com analgésicos e } \\
\text { AINEs e injeção intra-articular corticoanesté- } \\
\text { sica (com benefício sobretudo a curto prazo). } \\
\text { Na fase de rigidez: é fundamental a reabilitação } \\
\text { com fisioterapia. O TxC é eficaz na maioria dos } \\
\text { doentes. Outras opções terapêuticas mais re- } \\
\text { centes e em estudo, com evidência crescente } \\
\text { são terapia por ondas-choque extracorporal; } \\
\text { calcitonina e colagenases, distensão capsular } \\
\text { ecoguiada com ácido hialurónico, hidrodisten- } \\
\text { são capsular e distensão capsular artrográfica. } \\
\text { Perante falência do tratamento conservador } \\
\text { (após } 6 \text { meses) pode-se considerar o TxCx, sem- } \\
\text { pre seguido de reabilitação física. }\end{array}$ \\
\hline $\begin{array}{l}\text { - Artrose da } \\
\text { articulação } \\
\text { acromioclavicular }\end{array}$ & $\begin{array}{l}\text { Patologia degenerativa e } 2^{\text {a }} \text { doen- } \\
\text { ça mais comum no ombro em }>40 \\
\text { anos. Os doentes referem dor bem } \\
\text { localizada e estalos, cliques e rangi- } \\
\text { dos com o movimento. }\end{array}$ & $\begin{array}{l}\text { É clínico com a Rx como a imagem } \\
\text { recomendada para apoio ao diag- } \\
\text { nóstico. }\end{array}$ & $\begin{array}{l}\text { TxC com modificação da atividade, tratamento } \\
\text { de reabilitação (com fisioterapia), controlo álgi- } \\
\text { co farmacológico e infiltração cortico-anesté- } \\
\text { sica ecoguiada. O TxCx é considerado perante } \\
\text { ausência de melhoria com TxC após 3-6 meses. }\end{array}$ \\
\hline
\end{tabular}

AINE - Anti-inflamatório não esteroide; AVC - Acidente vascular cerebral; AVD - Atividade de vida diária; ECO - Ecografia; FR - Fatores de risco; RMN - Ressonância magnética; Rx - Radiografia; TC - Tomografia computorizada; TxC - Tratamento conservador; TxCx - Tratamento cirúrgico.

lar, sendo esta última a causadora da maioria dos casos de omalgia crónica. ${ }^{1,2,6}$

As patologias mais frequentes causadoras de omalgia são: patologia da coifa dos rotadores (incluindo a tendinopatia calcificante), instabilidade gleno-umeral, artrose gleno-umeral, capsulite adesiva e patologia da articulação acromioclavicular. ${ }^{1,2}$

De seguida, nas Tabelas 3, 4 e 5 resume-se a abordagem diagnóstica e terapêutica das principais etiologias intrínsecas de omalgia, por serem mais frequentes, não pretendendo esta descrição ser uma revisão exaustiva dessas patologias.

\section{CRITÉRIOS DE REFERENCIAÇÃO PARA CUIDADOS HOSPITALARES}

Os doentes devem ser referenciados para os cuidados de saúde secundários quando têm queixas álgicas não controladas com perturbação nas rotinas quotidianas (por exemplo, hábitos de sono) e disfunção significativa, apesar dos cuidados terapêuticos iniciais (por exemplo, reabilitação física); quando há confirmação ecográfica de rotura tendinosa da coifa dos rotadores sem meIhoria com tratamento conservador ao fim de 3 meses; quando há história de instabilidade ou dor articular, aguda e/ou pós-traumática; quando o diagnóstico não foi estabelecido no estudo inicial e estão presentes red flags mencionados previamente. , $^{1,7}$

\section{CONCLUSÃO}

A omalgia é um sintoma comum associado a morbilidade significativa. Os médicos de família têm a oportunidade de abordar, diagnosticar e orientar terapeuticamente este problema precocemente, enquanto o prognóstico é favorável. Com uma história clínica e exame objetivo completos, a maioria das patologias do ombro podem ser diagnosticadas numa primeira consulta e os planos de acompanhamento e terapêutica podem ser implementados com reduzidos meios de investigação laboratorial e imagiológica. Se esta última for necessária, a ecografia e a RMN são preferíveis à radiografia. No entanto, também é verdade que a radiografia continua a ser um 
TABELA 5. Abordagem diagnóstica e terapêutica das principais etiologias intrínsecas traumáticas de omalgia. 1,2,6,7

\begin{tabular}{|c|c|c|c|}
\hline PATOLOGIA & OBSERVAÇÕES & $\begin{array}{l}\text { MEIOS COMPLEMENTARES } \\
\text { DE DIAGNÓSTICO }\end{array}$ & TRATAMENTO \\
\hline \multicolumn{4}{|c|}{ PATOLOGIA INTRÍNSECA } \\
\hline \multicolumn{4}{|l|}{ Traumatologia } \\
\hline - Fratura da clavícula & $\begin{array}{l}\text { Mais comum nos < } 20 \text { anos. } O \\
\text { terço médio é o mais afetado. }\end{array}$ & $\begin{array}{l}\text { A Rx é o exame inicial; a TC permite melhor } \\
\text { caracterização de desvios, encurtamentos, } \\
\text { cominuição, extensão articular lesão vascu- } \\
\text { lar associada. }\end{array}$ & $\begin{array}{l}\text { Maioria, com TxC com suspensão bra- } \\
\text { quial simples ou um cruzado posterior, } \\
4-6 \text { semanas, coadjuvado com reabilita- } \\
\text { ção muscular adequada. São indicações } \\
\text { absolutas para TxCx (osteossíntese com } \\
\text { placa e parafusos): fratura aberta; risco } \\
\text { iminente de perfuração da pele; deterio- } \\
\text { ração neurológica e vascular progressi- } \\
\text { va; dissociação escapulo-torácica. }\end{array}$ \\
\hline $\begin{array}{l}\text { - Fratura do úmero } \\
\text { proximal }\end{array}$ & $\begin{array}{l}\text { Correspondem a } 45 \% \text { das fra- } \\
\text { turas do ombro. }\end{array}$ & $\begin{array}{l}\text { Estudo inicial com Rx. Para exclusão de fratu- } \\
\text { ras não visíveis na Rx ou melhor caracteriza- } \\
\text { ção das identificadas, realizar TC. }\end{array}$ & $\begin{array}{l}\text { 80\%- } 85 \% \text { são resolvidas com TxC, com } \\
\text { imobilização por } 3-4 \text { semanas com } \\
\text { suspensão braquial simples. As indica- } \\
\text { ções absolutas para TxCx são: fraturas } \\
\text { expostas, lesão neurovascular, fratura } \\
\text { patológica e ombro flutuante. }\end{array}$ \\
\hline $\begin{array}{l}\text { - Luxação da gleno- } \\
\text { umeral e instabilidade } \\
\text { articular traumática }\end{array}$ & $\begin{array}{l}\text { Podem ser luxações anterio- } \\
\text { res (maioria), posteriores, in- } \\
\text { feriores (raras) e superiores } \\
\text { (raras). } \\
\text { São emergências médicas e } \\
\text { devem ser reduzidas o mais } \\
\text { rapidamente possível. }\end{array}$ & $\begin{array}{l}\text { No diagnóstico deve ser realizado Rx. A ECO } \\
\text { é importante para excluir rotura da coifa a } \\
\text { partir dos } 60 \text { anos. A artro-RM é útil para } \\
\text { avaliar instabilidade gleno-umeral quando } \\
\text { doentes com }<40 \text { anos. }\end{array}$ & $\begin{array}{l}\text { Inicia-se com a redução da luxação e } \\
\text { avaliação da integridade do nervo axilar. } \\
\text { É essencial a suspensão braquial } 2-3 \text { se- } \\
\text { manas e TxF. O TxCx deve ser ponderado } \\
\text { e adaptado às lesões encontradas e ao } \\
\text { tipo de doente. }\end{array}$ \\
\hline $\begin{array}{l}\text { • Luxação } \\
\text { acromioclavicular }\end{array}$ & $\begin{array}{l}\text { Patologia comum em jovens, } \\
\text { sobretudo no sexo masculi- } \\
\text { no. É classificada em } 6 \text { tipos } \\
\text { (I a VI) consoante o padrão de } \\
\text { rotura dos ligamentos coraco- } \\
\text { clavicular e acromioclavicular } \\
\text { e na distância coracoclavicu- } \\
\text { lar. }\end{array}$ & $\begin{array}{l}\text { É recomendada a Rx e é fundamental a com- } \\
\text { paração com o lado não afetado. }\end{array}$ & $\begin{array}{l}\text { O TxC (crioterapia, analgesia farmaco- } \\
\text { lógica e suspensão braquial por } 3 \text { sema- } \\
\text { nas) está indicado nas luxações tipo I, II e } \\
\text { na maioria das III. } \\
\text { O TxCx está indicado nas luxações tipo } \\
\text { IV, V e VI. }\end{array}$ \\
\hline
\end{tabular}

ECO - Ecografia; RM - Ressonância magnética; Rx - Radiografia; TC - Tomografia computorizada; TxC - Tratamento conservador; TxCx - Tratamento cirúrgico; TxF - Tratamento fisiátrico.

importante método de screening (para deteção de calcificações, artrose e conflito subacromial), especialmente se associada à ecografia articular; ao contrário da RMN, que pela limitação de requisição nos cuidados de saúde primários, deve ser reservada para as consultas hospitalares de especialidade do ombro, para diagnósticos mais complexos e relacionados com patologia intra-articular (por exemplo nos jovens com sinais de instabilidade para despiste de lesões labrais) e na definição de critérios de cirurgia nos doentes com rotura da coifa. A referenciação a cuidados de saúde secundários (Ortopedia, Reumatologia, Medicina Interna) deve ser levada a cabo, quando as queixas são refratárias à abordagem inicial.

\section{RESPONSABILIDADESÉTICAS}

CONFLITOS DE INTERESSE: Os autores declaram não possuir conflitos de interesse.

SUPORTE FINANCEIRO: O presente trabalho não foi suportado por nenhum subsídio ou bolsa.

PROVENIÊNCIA E REVISÃO POR PARES: Não comissionado; revisão externa por pares.

\section{ETHICAL DISCLOSURES}

CONFLICTS OF INTEREST: The authors have no conflicts of interest to declare.

FINANCIAL SUPPORT: This work has not received any contribution grant or scholarship.

PROVENANCE AND PEER REVIEW: Not commissioned; externally peer reviewed.

\section{REFERÊNCIAS}

1. Vaz R, Silva AJ, Cordeiro A, Aires A, Gonçalves AF, Costa AJ, et al. Patologia Musculo-esquelética para médicos de família. Lisboa: Heartbrain - Consultores em Comunicação Lda; 2021.

2. House J, Mooradian A. Evaluation and management of shoulder pain in primary care clinicians. South Med J. 2010;103:112935. doi: 10.1097/SMJ.0b013e3181f5e85f.

3. Masters S, Burley S. Shoulder pain. Aust Fam Physician. 2007;36:414-6, 418-20.

4. Mitchell C,AdebajoAde, Hay Elaine, Carr A.Shoulderpain:diagnosis and management in primary care. BMJ. 2005;331:11248. doi: 10.1136/bmj.331.7525.1124.

5. Teixeira DC, Alves L, Gutierres M. The role of scapular dyskinesis on rotator cuff tears: a narrative review of the current knowledge. EFORT Open Rev. 2021;6:932-40. doi: 10.1302/2058-5241.6.210043. 
6. Vaughan A, Hulkower S. Evaluation of the adult with shoulder complains. UpToDate. [Acedido a 31 de julho de 2021]. Disponível em: https://www.uptodate.com/contents/evaluation-of-the-adult-with-shoulder-complaints.

7. Cardoso A, Branco JC, Silva JP, Cruz M, Costa MM. Regras de Ouro em Reumatologia. Lisboa: Direção-Geral da Saúde; 2005.

8. Simons SM, Dixon JB. Physical examination of the shoulder. UpToDate. [Acedido a 31 de julho de 2021]. Disponível em: https://www.uptodate.com/contents/physical-examination-of-the-shoulder.

9. Silva R, Pimentel A, Gutierres M. Calcific Tendinopathy of the Rotator Cuff. Current Solutions. Prog Orthop Sci. 2020;6:1-8. doi: 10.47363/POS/20201004

10. Silva R, Pimentel A, Gutierres M. A literature review of the treatment options for Idiopathic Adhesive Capsulitis of the Shoulder. Orthop Spo Med Op Acc J. 2021;4:460-8. doi: 10.32474/OSMOAJ.2021.04.000199. 